\title{
Evaluating Co-Occurrence of GOF Design Patterns with God Class and Long Method Bad Smells
}

\author{
Bruno L. Sousa \\ UFMG - ICEx \\ Department of Computing \\ Belo Horizonte, MG - Brazil \\ bruno.luan.sousa@dcc.ufmg.br
}

\author{
Mariza A. S. Bigonha \\ UFMG - ICEX \\ Department of Computing \\ Belo Horizonte, MG - Brazil \\ mariza@dcc.ufmg.br
}

\author{
Kecia A. M. Ferreira \\ CEFET-MG \\ Department of Computing \\ Belo Horizonte, MG - Brazil \\ kecia@decom.cefetmg.br
}

\begin{abstract}
Design patterns are general reusable solutions to common recurring problems in software projects. These solutions, when correctly applied, are supposed to enhance modular and flexible structures in software. The aim of this work is to study the occurrences of God Class and Long Method bad smells in software systems developed with design patterns. To achieve this aim, we carried out a study with five Java project, in order to: (i) investigate if the use of GOF design patterns avoid the occurrences of the bad smells God Class and Long Method, (ii) identify co-occurrences of the GOF design patterns with these bad smells, and (iii) identify the main situations that lead software systems to present these co-occurrences. The results obtained suggest that Composite and Factory Method have a low co-occurrence with these bad smells, and Template Method and Observer have a high co-occurrence with God Class and Long Method, respectively. In addition, we have identified that the misuse of design patterns and the scattering and crosscutting concerns has contributed to the emergence of such co-occurrences.
\end{abstract}

\section{CCS Concepts}

-General and reference $\rightarrow$ Measurement; •Software and its engineering $\rightarrow$ Design patterns; •Social and professional topics $\rightarrow$ Quality assurance;

\section{Keywords}

Design Patterns, Bad Smells, Software Metrics, Thresholds.

\section{INTRODUCTION}

Design pattern is a general solution to a recurring problem in a given context in the software design [9]. Its main goal is to create flexible and extensible software systems, with a reusable structure and easy maintenance. They are recognized as good programming practice, which, when applied correctly, may help reducing bad smells in software,

Permission to make digital or hard copies of all or part of this work for personal or classroom use is granted without fee provided that copies are not made or distributed for profit or commercial advantage and that copies bear this notice and the full citation on the first page. To copy otherwise, or republish, to post on servers or to redistribute to lists, requires prior specific permission and/or a fee.

SBSI 2017 June $5^{\text {th }}-8^{\text {th }}$, 2017, Lavras, Minas Gerais, Brazil

Copyright SBC 2017. although they have not been proposed for this purpose [15]. Bad smells, according to Fowler and Beck [8], are symptoms presented in the source code of a program that possibly indicate a more serious problem that requires code refactoring [8]. Regions of code that exhibit these symptoms are not considered errors, but they impair software quality and violate Software Engineering principles such as modularity, readability and reuse. Design patterns may be used to remove bad smells. On the other hand, there are studies that identify co-occurrence of design patterns and bad smells [3, $10,11,18]$. Although design patterns are intended to improve software quality, they do not necessarily avoid bad smells.

The goal of this work is to investigate object-oriented projects that apply the design patterns defined by Gamma et al. (GOF catalog) [9], and, using a methodology based on extraction of metrics, answer the research questions:

- RQ1: Do the design patterns defined in the GOF catalog avoid the occurrence of God Class and Long Method bad smells in software?

- RQ2: Which design patterns of GOF catalog presented co-occurrence with the God Class and Long Method bad smells?

- RQ3: What are the more common situations in which the God Class and Long Method bad smells appear in software systems that apply GOF design patterns?

In order to answer these research questions, a case study with eleven of the twenty-three design patterns described by Gamma et al. [9] and five Java based software systems from the Qualitas.class Corpus [16] was carried out. These software systems make use of design patterns.

The main finding of this work is that design patterns, when implemented without considering good programming practices, may generate situations that lead to the presence of bad smells. These situations need to be identified, so that, the software may be refactored in order to eliminate the problematic structures.

\section{RESEARCH METHODOLOGY}

This study was carried out in 5 steps: (i) identification of bad smells detection strategies, (ii) definition of the data set that comprises the software systems considered in the study, (iii) data collection, (iv) application of the association rules and (v) method of the data analysis. 


\subsection{Identification of Bad Smells Detection Strategies}

According to Marinescu [13], detection strategy is a quantifiable expression of a rule that evaluates if fragments of a source code have properties of a given bad smell. In addition to detection strategies, software metric thresholds can be used to determinate the relationship of a metric with a bad smell and, thus, identify anomalous entities.

In this study, we consider the God Class and Long Method bad smells. Lanza and Marinescu [12] describe God Class as a class that executes too much work and delegate minor details to other classes. Fowler and Beck [8] describe the Long Method bad smell as a method that performs too much work, having many lines, temporary variables and parameters. We choose these bad smells because they are especially problematic to the software maintenance and they are related to a large amount of information that may turn the software comprehension hard and increase coupling among the methods and among the classes of the system.

The detection strategies used in this study were proposed by Filó et al. [7]. We choose these strategies because they are composed of well known software metrics. Besides that, they were previously evaluated by Filó [5], and no false negative was returned. False positive may be returned, but with a low probability of occurrence. These results suggest that these detection strategies are effective in bad smells detection. The thresholds defined by Filó et al. [7] for a software metric is classified in three ranges: Good, Regular, and Bad. The experiments done by the authors indicate that the Good range is related to low occurrences of bad smells. Therefore, the detection strategies rely on the Regular and Bad ranges of the metric values. Following, we describe the detection strategies of Filó et al. [7] for God Class and Long Method.

The detection strategy of bad smell God Class, Figure 1, uses the metrics Weighted Methods per Class (WMC), Number of Methods (NOM), Number of Attributes (NOF), and Lack of Cohesion of Methods (LCOM).

The detection strategies for the Long Method bad smell, Figure 2, uses the metrics Method Lines of Code (MLOC), Nested Block Depth (NBD) and McCabe Cyclomatic Complexity (VG).

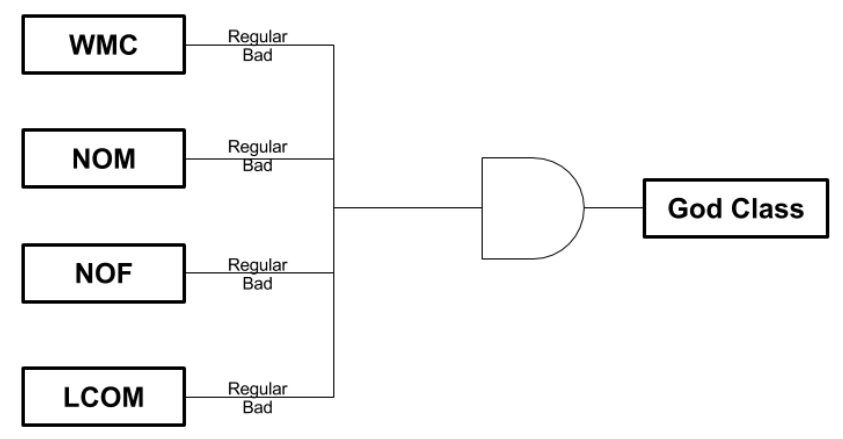

Figure 1: Detection strategy for God Class extracted from [7].

\subsection{Data Set}

The software sample used in this study are from Qualitas.class Corpus [16], a data set which comprises software metrics of 112 open source software systems developed in

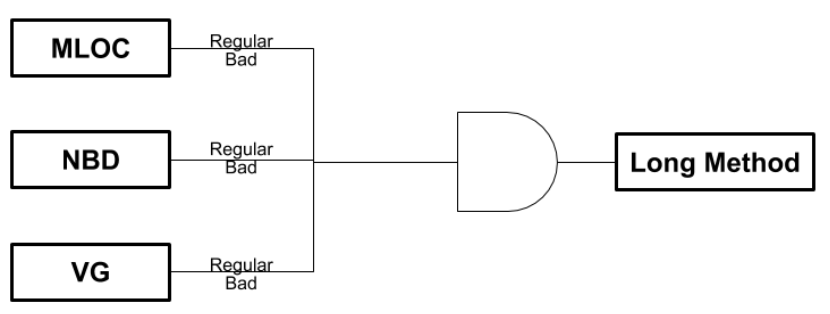

Figure 2: Detection strategy for Long Method extracted from [7].

Java. Qualitas.class Corpus provides 23 software metrics and the bytecodes of the software systems. This data set was chosen because it has a large collection of open source projects developed in Java that are widely used in empirical studies of software artifacts.

As this study involves manual inspection, we considered a sample of five software systems: Hibernate 4.2.0, JHotDraw 7.5.1, Kolmafia 17.3, Webmail 0.7.10 and Weka 3.6.9. All of them, except Kolmafia, are from Qualitas Corpus. The main criteria for the selection of these project was based in two points, they use design patterns from GOF catalog and they present the bad smells considered in this work. It was decided to include Kolmafia 17.3 in this sample because previous studies to point out values of metrics considered problematic in this software system [4], however, without any correlation of these values with bad smell or design patterns.

\subsection{Data Collection}

The third step involved the collection of the data analyzed in this work. Qualitas.class Corpus comprises files in XML format with the software metrics of the software systems. As most of the software used in this work are from Qualitas.class Corpus, these files were used. Since Kolmafia 17.3 data are not in this corpus, its code was downloaded and its metrics was collected. The tools Eclipse 4.2 Juno IDE ${ }^{1}$ and Metrics 1.3.6 plugin $^{2}$ were used for this purpose. The metrics of Kolmafia 17.3 were exported to XML file.

To verify the existence of design patterns in the software, a tool proposed by Tsantsalis et al. [17], called Design Pattern Detection using Similarity Scoring 4 (DPDSS), was used. According to the authors, this tool models all aspects of design patterns by means of directed graphs, represented by quadratic matrices, and applies an algorithm called Similarity Scoring. This algorithm receives as input the system and the graph of the design pattern, and then, calculates the similarity scores between the vertices of the graph. According to Tsantsalis et al. [17], the main advantage of this approach is the ability to detect not only the patterns in their base form, which is normally found in the literature, but also the modified versions of it. We previously tested DPDSS with three systems: JHotDraw 5.1, JRefactory 2.6.24 and JUnit 3.7 , and no false positive occurrence of design patterns was returned. False negatives were returned only for two design patterns: Factory Method and State. The results presented by this tool were very satisfactory, showing that it is effective

\footnotetext{
${ }^{1}$ http://www.eclipse.org/downloads/packages/release/ Juno/SR2

${ }^{2}$ http://metrics.sourceforge.net
} 
in identifying instances of design patterns.

Filó et al. [6] developed a tool, RAFTool, which performs the identification of methods, classes and packages with anomalous measurements of object oriented software metrics. RAFTool was used with the purpose of implementing detection strategies. The tool receives as entry the XML of the target system, with its software metrics, and a detection strategy that is described by a logical expression in a given format. The tool reports the classes or the methods whose metric values fit to the detection strategy.

In RAFTool, the metrics' thresholds that comprise the detection strategy are represented by the following keywords: COMMON, which corresponds to the Good/Frequent ranges of the metrics, CASUAL, which corresponds to the REGULAR/OCCASIONAL ranges of the metrics, and UNCOMMON, which corresponds the Bad/Rare ranges of the metrics. The logical expression of God Class and Long Method are defined as follows.

\section{Exp1 ( UNCOMMON[WMC] OR CASUAL[WMC] ) AND ( UNCOMMON[NOF] OR CASUAL[NOF] ) AND ( UN- COMMON[NOM] OR CASUAL[NOM] ) AND ( UNCOM- MON[LCOM] OR CASUAL[LCOM] ) \\ Exp2 ( UNCOMMON[MLOC] OR CASUAL[MLOC] ) AND ( UNCOMMON[NBD] OR CASUAL[NBD] ) AND ( UN- COMMON[VG] OR CASUAL[VG] )}

Instances of design patterns returned by DPDSS can be composed of one or more classes or methods. An example of this is the instance returned to the Bridge design pattern that has a class responsible for representing the implementation part and a class responsible for representing the abstraction part. To solve this problem, we developed the Design Pattern Smell ${ }^{3}$ [14] to count the classes and the methods in the instances of a design pattern, as well as to identify the artifacts, classes or methods, that have a given design pattern and a given bad smell. This tool receives as entry (1) the files in the XML format exported by DPDSS, containing the instances of design patterns of a software system, and (2) the CSV files generated by RafTool, containing the artifacts with a given bad smell.

\subsection{Application of Association Rules}

To identify the co-occurrences of bad smells and design patterns we applied association rules, based on concepts of data mining $[1,2]$. We decided to use the association rules because they combine items from a data set to extract knowledge about the data. Moreover, previous works in the same context of this one [3, 18] have applied association rules.

To apply the association rules, three metrics are used: Support [1], Confidence [1], and Conviction [2]. These metrics are based in the following main concepts: Transaction, defined as a set of items; Antecedent that is an item that appears on the left side of the association rule; and Consequent, an item that appears on the right side of the association rule. Therefore, a basic association rule has the following form: Antecedent $\Rightarrow$ Consequent.

Support (sup) of an association rule corresponds to the frequency that an item occurs in a transaction (Equation 1).

\footnotetext{
${ }^{3}$ http://www2.dcc.ufmg.br/laboratorios/llp/Products/ indexProducts.html
}

For instance, let us consider a shopping base in a supermarket. Suppose that there is a data set with 1,000 transactions, which are the set of items that were purchased. In this data set, the items pasta and tomato appear together in 100 records. So, Support for this relationship is 0.1, i.e., $10.0 \%$.

$$
\sup (X \Rightarrow Y)=P(X, Y)
$$

Confidence (conf) expresses the probability of a Consequent occurs since Antecedent has occurred (Equation 2).

$$
\operatorname{con} f(X \Rightarrow Y)=\frac{\sup (X \Rightarrow Y)}{\sup (X)}
$$

In the aforementioned example, let us consider that the item pasta is found alone in 200 of 1,000 transactions of the data set. To compute the Confidence of the association rule pasta $\Rightarrow$ tomato, it is necessary to divide the Support of this rule, 0.1 , by the Support of pasta - Antecedent in the association rule,- 0.2 , resulting in a confidence of 0.5 , i.e., $50.0 \%$. Confidence is very sensitive to the frequency on the right side of the association rule, i.e., a very high value in the right side of the association rule can generate a high confidence value, even if the items do not have any type of relation.

To solve this problem of Confidence, Brin et al. [2] proposed the metric Conviction. This metric uses the Support in both the Antecedent and the Consequent (Equation 3).

$$
\operatorname{conv}(X \Rightarrow Y)=\frac{\sup (X) *(1-\sup (Y))}{\sup (X)-\sup (X \Rightarrow Y)}
$$

In the given example, let us consider that the item tomato is found alone in 300 of 1,000 transactions of the data set. Thus, the support tomato, sup(tomato) is 0.3 and the confidence $\operatorname{conf}($ pasta $\Rightarrow$ tomato) is 0.5 . Applying these values in the Equation 3, the Conviction conv(pasta $\Rightarrow$ tomato) is 1.4. When the value of Conviction is 1.0 , it indicates that the antecedent and the consequent have no relation at all. When the value of Conviction value is less than 1.0, it indicates that if the antecedent occurs, the consequent tends to not occur. When the value of the Conviction is greater than 1.0 , it means that the antecedent and the consequent have relation; the greater the value of Conviction, the greater the relation between the antecedent and the consequent. An infinite result indicates that the antecedent never appears in the transactions.

In this work, for the application of the association rules, a transaction represents each class in the analyzed system; antecedent represents a design pattern; consequent represents a bad smell, in particular, the bad smells God Class and Long Method.

\subsection{Method of the Data Analysis}

Figure 3 illustrates, via diagram, the method used to analyze the data obtained in the study.

This step is made in 5 parts. Initially, we identify the design patterns in the software systems. In order to identify them, the tool DPDSS was used and the results obtained were stored in a table.

The second part aims to identify classes and methods that have the God Class or Long Method bad smells. To find such methods and classes, we applied the logical expressions 


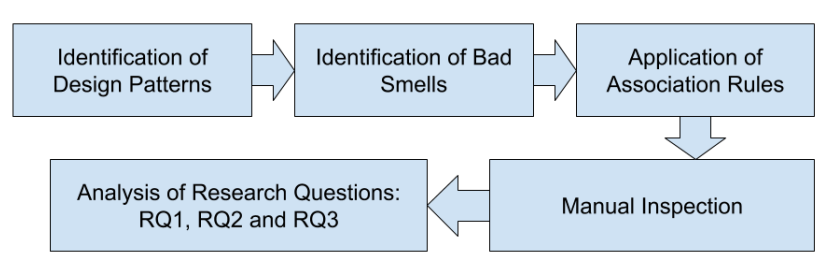

Figure 3: Method of the data analysis.

Exp1 and Exp2 in RAFTool. Once the design patterns and the God Class and Long Method bad smells were identified in the software projects, in the sequel, the pre-processing of these data was performed by running the Design Pattern Smell tool. In the third part, we apply the association rules on the data resulting from the pre-processing, and then, we identify the co-occurrences existing in the software systems. In the fourth part, we manually inspected the classes with co-occurrence, in order to identify the situations that favored the presence of this relation in such classes. In the fifth part, the data were analyzed in order to answer the research questions of this work.

\section{RESULTS}

This section presents the results followed by the discussion of the presented study, as well as the answers to the proposed research questions.

Tables 1 and 2 show the amount of classes and methods of the software systems with the bad smells God Class and Long Method, respectively. All systems, in some level, presented these bad smells.

\begin{tabular}{|l|r|r|r|}
\hline Software & $\begin{array}{c}\text { \# Classes with } \\
\text { God Class }\end{array}$ & \# Classes & $\begin{array}{c}\text { \% Classes with } \\
\text { God Class }\end{array}$ \\
\hline Hibernate & 527 & 7,711 & $6.83 \%$ \\
\hline JHotDraw & 122 & 1,061 & $11.50 \%$ \\
\hline Kolmafia & 385 & 3,225 & $11.94 \%$ \\
\hline Webmail & 15 & 129 & $11.63 \%$ \\
\hline Weka & 467 & 2,401 & $19.45 \%$ \\
\hline
\end{tabular}

Table 1: Amount of classes with God Class.

\begin{tabular}{|l|r|r|r|}
\hline Software & $\begin{array}{c}\text { \# Methods with } \\
\text { Long Method }\end{array}$ & \# Methods & $\begin{array}{c}\text { \% Methods with } \\
\text { Long Method }\end{array}$ \\
\hline Hibernate & 2,883 & 48,234 & $5.98 \%$ \\
\hline JhotDraw & 995 & 7,633 & $13.04 \%$ \\
\hline Kolmafia & 5,400 & 2,8078 & $19.23 \%$ \\
\hline Webmail & 131 & 1,091 & $12.01 \%$ \\
\hline Weka & 3,822 & 20,871 & $18.31 \%$ \\
\hline
\end{tabular}

Table 2: Amount of methods with Long Method.

Tables 3 and 4 show the results after pre-processing the data. In both tables, the "T" column indicates the total number of classes or method that uses a design pattern, and the "DP\&BS" column indicates the number of classes that have instances of some design pattern (DP) and occurrence of the respective bad smell (BS).

After pre-processing the data, the application of the association rule is performed, in order to identify the co-occurrences. For this purpose, the metrics described in Section 2.4 were calculated using the results of Tables $1,2,3$, and 4 . We used the result of the Conviction metric following the thresholds shown in Section 2.4. Figure 4 exhibits the results of the
Conviction metric for the God Class bad smell, considering the association rule Design Pattern $\Rightarrow$ God Class. Figure 5 shows the results of the same metric for Long Method bad smell, according to the association rule Design Pattern $\Rightarrow$ Long Method.

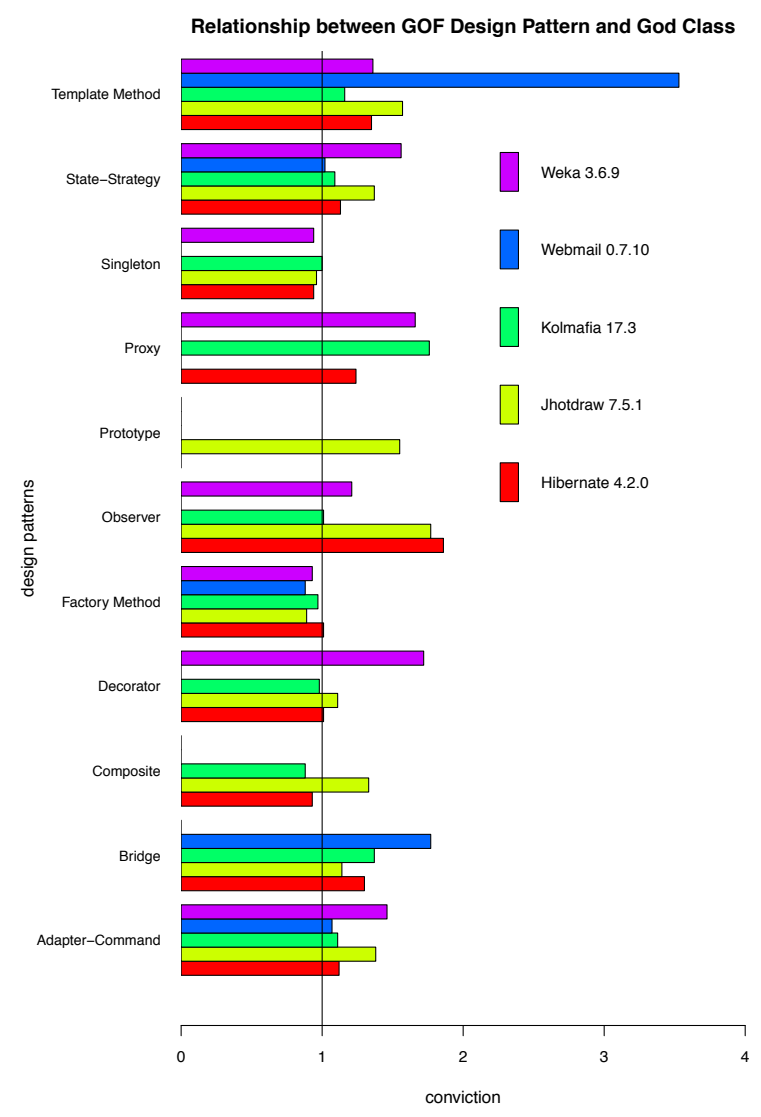

Figure 4: Results of the association rule Design Pattern $\Rightarrow$ God Class.

\subsection{Analysis of the Results}

The classes with the God Class or Long Method bad smells were inspected manually in order to answer the research questions defined in this work.

RQ1. Do the design patterns defined in the GOF catalog avoid the occurrence of God Class and Long Method bad smells in software?

The results reported in Table 3 indicate that two design patterns have a low occurrence of God Class: Composite and Factory Method. The manual inspection reveals that they have a modular structure that divides the tasks between several classes. The idea of the Composite pattern is to construct complex objects by means of simpler objects. These simpler objects are defined in modules, in such a way intelligence is divided between them, reducing the complexity of the classes. The Factory Method pattern simulates the idea of a factory in which there is an interface to create objects, but the creation of the object itself occurs in the class that implements that interface. Thus, it is possible to create several modules, each one responsible for creating 
XIII Brazilian Symposium on Information Systems, Lavras, Minas Gerais, June 5-8, 2017

\begin{tabular}{|c|c|c|c|c|c|c|c|c|c|c|}
\hline \multirow[t]{2}{*}{ Design Pattern } & \multicolumn{2}{|c|}{$\begin{array}{c}\text { Hibernate } \\
4.2 .0\end{array}$} & \multicolumn{2}{|c|}{$\begin{array}{c}\text { JHotDraw } \\
7.5 .1\end{array}$} & \multicolumn{2}{|c|}{$\begin{array}{c}\text { Kolmafia } \\
17.3\end{array}$} & \multicolumn{2}{|c|}{$\begin{array}{c}\text { Webmail } \\
0.7 .10\end{array}$} & \multicolumn{2}{|c|}{$\begin{array}{c}\text { Weka } \\
3.6 .9\end{array}$} \\
\hline & $\mathrm{T}$ & $\mathrm{DP} \& \mathrm{BS}$ & $\mathrm{T}$ & $\mathrm{DP} \& \mathrm{BS}$ & $\mathrm{T}$ & DP\&BS & $\mathrm{T}$ & DP\&BS & $\mathrm{T}$ & DP\&BS \\
\hline Adapter-Command & 228 & 39 & 53 & 19 & 386 & 81 & 40 & 7 & 152 & 68 \\
\hline Bridge & 56 & 16 & 40 & 9 & 14 & 5 & 6 & 3 & 0 & 0 \\
\hline Composite & 12 & 0 & 12 & 4 & 8 & 0 & 0 & 0 & 0 & 0 \\
\hline Decorator & 37 & 3 & 10 & 2 & 67 & 7 & 0 & 0 & 32 & 17 \\
\hline Factory Method & 37 & 3 & 5 & 0 & 31 & 3 & 2 & 0 & 22 & 3 \\
\hline Observer & 4 & 2 & 2 & 1 & 8 & 1 & 0 & 0 & 36 & 12 \\
\hline Prototype & 0 & 0 & 21 & 9 & 0 & 0 & 0 & 0 & 0 & 0 \\
\hline Proxy & 8 & 2 & 0 & 0 & 18 & 9 & 0 & 0 & 35 & 18 \\
\hline Singleton & 232 & 3 & 13 & 1 & 77 & 9 & 1 & 1 & 34 & 5 \\
\hline State-Strategy & 271 & 47 & 121 & 43 & 334 & 64 & 23 & 3 & 93 & 45 \\
\hline Template Method & 87 & 27 & 16 & 7 & 54 & 13 & 4 & 3 & 22 & 9 \\
\hline
\end{tabular}

Table 3: Amount of classes that comprise each design pattern and amount of classes that contain both design pattern and the bad smell God Class.

\begin{tabular}{|c|c|c|c|c|c|c|c|c|c|c|}
\hline \multirow[t]{2}{*}{ Design Pattern } & \multicolumn{2}{|c|}{$\begin{array}{c}\text { Hibernate } \\
4.2 .0\end{array}$} & \multicolumn{2}{|c|}{$\begin{array}{c}\text { JHotDraw } \\
7.5 .1\end{array}$} & \multicolumn{2}{|c|}{$\begin{array}{c}\text { Kolmafia } \\
17.3\end{array}$} & \multicolumn{2}{|c|}{$\begin{array}{c}\text { Webmail } \\
0.7 .10\end{array}$} & \multicolumn{2}{|c|}{$\begin{array}{c}\text { Weka } \\
3.6 .9\end{array}$} \\
\hline & $\mathrm{T}$ & DP\&BS & $\mathrm{T}$ & DP\&BS & $\mathrm{T}$ & DP\&BS & $\mathrm{T}$ & DP\&BS & $\mathrm{T}$ & DP\&BS \\
\hline Adapter-Command & 271 & 52 & 73 & 19 & 703 & 123 & 50 & 5 & 222 & 71 \\
\hline Bridge & 61 & 13 & 51 & 11 & 19 & 3 & 8 & 2 & 0 & 0 \\
\hline Composite & 8 & 0 & 29 & 0 & 37 & 0 & 0 & 0 & 0 & 0 \\
\hline Decorator & 115 & 2 & 31 & 1 & 255 & 12 & 0 & 0 & 61 & 25 \\
\hline Factory Method & 58 & 0 & 23 & 0 & 45 & 0 & 2 & 0 & 27 & 0 \\
\hline Observer & 8 & 3 & 2 & 1 & 7 & 3 & 0 & 0 & 24 & 4 \\
\hline Prototype & 0 & 0 & 16 & 6 & 0 & 0 & 0 & 0 & 0 & 0 \\
\hline Proxy & 6 & 1 & 0 & 0 & 31 & 9 & 0 & 0 & 37 & 12 \\
\hline Singleton & 340 & $\overline{0}$ & 15 & 0 & 672 & 0 & 1 & 0 & 83 & 0 \\
\hline State-Strategy & 343 & 121 & 227 & 90 & 974 & 154 & 19 & 0 & 173 & 97 \\
\hline Template Method & 275 & 90 & 47 & 13 & 161 & 48 & 14 & 3 & 34 & 16 \\
\hline
\end{tabular}

Table 4: Amount of methods that comprise each design pattern and amount of methods that contain both design pattern and the bad smell Long Method.

and managing the information of a set of objects in the system, removing the workload from a single class. Therefore, both Composite and Factory Method are design patterns intrinsically modular.

A similar behavior is observed for the Long Method bad smell, as shown in Table 4. Most design patterns present a high amount of occurrences of Long Method, except Composite and Factory Method, which present just a few occurrences of this bad smell.

The Singleton design pattern is a special case. Although its instances do not have the Long Method bad smell, it seems a false negative case. Singleton was indicated as false negative because the instances of this design pattern identified by DPDSS are based only on the static attribute presented in the class. It does not consider methods as characteristic of this design pattern. For this reason, when this design pattern was intersected with methods that had bad smells, it returned 0. However, when classes containing Singleton were inspected, we identified methods with the Long Method bad smell inside them.

Although Factory Method and Composite design patterns present few co-occurrences with the God Class and Long Method bad smells, the general conclusion of this analysis is that most of the design patterns studied in this work are associated with this two bad smells. Therefore, the answer of RQ1 is "No, design patterns GOF not necessarily avoid occurrences of the God Class and Long Method bad smells."

RQ2. Which design patterns of GOF catalog presented cooccurrence with the God Class and Long Method bad smells?

In order to analyze the associations between design patterns and bad smells, we considered the values of Conviction. The choice of this metric is due to the fact that Conviction is able to establish a relationship between Support and Confidence metrics. Moreover, Conviction has a better sensitivity to the direction between the antecedent and the consequent. In order to define which co-occurrences of design patterns and bad smells have the highest relation, the thresholds of the Conviction, mentioned in Section 2.4, were considered.

The chart in Figure 4 indicates a strong relationship of several design patterns with the bad smell God Class. The design patterns with the highest Conviction values are: Template Method, Observer, and Proxy. We also observed that the results for those design patterns in four systems are very close: Hibernate 4.2.0, JHotdraw 7.5.1, Kolmafia 17.3, and Weka 3.6 .9. In Webmail 0.7.10, the association rule Template Method $\Rightarrow$ God Class prevailed over the others, showing that the Template Method was the design pattern that presented more co-occurrences with God Class. These results, then, indicate that Template Method, Observer, and 




Figure 5: Results of the association rule Design Pattern $\Rightarrow$ Long Method.

Proxy presented more occurrences of the bad smell God Class than the other design patterns.

Long Method has a similar behavior of God Class. The chart in Figure 5 shows that many design patterns have a strong relation with Long Method. Three design patterns present a higher co-occurrence with Long Method: Observer, State-Strategy, and Template Method. Conviction is higher in 3 out of 5 systems: Hibernate 4.2.0, JHotDraw 7.5.1, and Kolmafia 17.3. The strongest association rule in these systems is Observer $\Rightarrow$ Long Method. Webmail 0.7.10 does not present any instance of Observer, therefore the association rule Observer $\Rightarrow$ Long Method was not found in this project. In Weka 3.6.9, Observer $\Rightarrow$ Long Method has a low occurrence. In conclusion, the association rule Observer $\Rightarrow$ Long Method, despite having a low occurrence in two cases, was the one that presented the strongest Conviction in this study. Nevertheless, State-Strategy and Template Method also present a high Conviction value. Then, the results indicate that Observer, State-Strategy, and Template Method presented more occurrences of the Long Method bad smell than the other design patterns.

So, answering RQ2, Template Method, Observer and Proxy were identified as those who presented the main co-occurrences with the God Class bad smell with Template Method have the highest co-occurrence with this bad smell. The Observer, State-Strategy and Template Method design patterns were identified as those that presented the main co- occurrences with the Long Method bad smell. Nevertheless, Observer presented the highest co-occurrence with Long Method.

RQ3. What are the more common situations in which the God Class and Long Method bad smells appear in software systems that apply GOF design patterns?

The results found in this study indicate that God Class bad smell has more co-occurrences with the Template Method design pattern, and the Long Method bad smell has more co-occurrences with the Observer design pattern. In order to identify the causes of such co-occurrences, a manual inspection was performed in the classes that present the relations Template Method $\Rightarrow$ God Class and Observer $\Rightarrow$ Long Method.

Template Method aims to define the skeleton of an algorithm via an operation, transferring some steps to the subclasses, which have the power to redefine the characteristics of the algorithm without changing the algorithm structure [9]. That is, this pattern uses a modular structure, in which the various behaviors of an object are modeled in the subclasses and assigned to the object via polymorphism. The advantage of this implementation is the reduction of complexity in the super class, since definitions via conditional structures such as if, else, and switches are replaced by polymorphism. However, using this pattern requires careful attention to avoid assigning many responsibilities to the templates and also to the super class.

The manual inspection in the classes that presented cooccurrence of Template Method $\Rightarrow$ God Class indicated that a great amount of responsibilities were assigned to the classes containing the template method. It was observed that the templates methods refer to the definition of the behavior of the object, implemented in the subclasses. In some classes, the implementation of behavior has a high complexity. This generates an overload of tasks in the templates methods, contributing with the occurrence of Long Method in some cases. In addition, a high number of dependencies has been observed in super classes that implements a Template Method. Several objects are instantiated in such implementations, elevating the coupling of these classes, and small tasks are passed on to them. In these classes there are an intense amount of getter methods that make use of few attributes.

Based on this analysis, it is possible to identify some reasons that contributed to the co-occurrence of Template Method $\Rightarrow$ God Class. The Template Method design pattern allows the extension of classes and the addition of new features. However, poor planning and misapplication of this design pattern contributes to increase the amount of superclass responsibilities, concentrating a large part of the intelligence of the system in the super-class, generating its cooccurrence with God Class bad smell. To eliminate these cooccurrences, it is necessary to extract, from the overloaded classes, methods and attributes, adding them to other classes, in order to divide the amount of work and effort performed by the Template classes.

Observer is a solution that establishes a one-to-many dependency between objects. Observer uses a structure where the subject class has a list of all observers classes that use its data. When some information is changed in the subject by one of its observers, the subject is triggered by changing the other observers. The aim of this design pattern is the synchronization of data and the updating of objects in real 
time. This update occurs via polymorphism, avoiding the increase of complexity that generally occurs with the use of conditional structures. However, when using this design pattern, it is important to implement the operation that notifies the observers in the subject properly, since a poor planning of this operation can result in complex methods.

The methods involved in the relation Observer $\Rightarrow$ Long Method, were manually inspected. It was noticed that the methods implemented in the subject class, responsible for notifying observers, perform a lot of work. Such methods are big and complex, what make the code difficult to read and to understand. In some case, there is also scattering in the code, involving log records and observer update, among others, that influence the high complexity and the large size of these methods.

The aforementioned analysis revealed some main situations that may lead to the presence of the Long Method bad smell in methods implemented in Observer. The high amount of code repetitions and responsibilities assigned to the notifying observers methods are the main situation we have found. Scattering and crosscutting concerns also have appeared in the implementation of such methods. A concern is a part of a problem that one needs to deal with in a software system. The registration of the operation log is an example of concern. When concerns are not modularized in a program, it leads to scattering and to crosscutting concerns in the code. In the methods implemented in Observer, we have detected scattering and crosscutting concerns in subject classes, specially due to repetition of code that implements register of $\mathrm{log}$, aiming to notify observers. To eliminate such co-occurrences, it is necessary to eliminate these code repetitions and to modularize them so that they are implemented in a single entity and can be reused by other entities. With respect to scattering and crosscutting concerns, in object-oriented programming, these occurrences are difficult to control. However, they can be mitigated through the Singleton design pattern. Developers could create a Singleton class, responsible for managing log concern for example, that would provide a specific method for logging, requesting only the information that should be written.

In conclusion, the main reason of co-occurrences of God Class bad smell with Template Method is the misuse of object orientation, leading to concentration of responsibilities in the classes that implements the Template Method. A similar situation was also found in the case of the co-occurrences of Long Method bad smell with Observer; in this case, the misuse of object orientation leads to scattering and crosscutting concerns.

\section{THREATS TO VALIDITY}

In this section, we discuss the main threats to the validity of this work. We considered a sample composed of five software systems in this study. We mainly analyzed systems from a large data set, called Qualitas Corpus. The sample has small, medium, and large systems. Nevertheless, due to the small size of the sample, we are not able to generalize the results found in this study. Even though, the results found are still important because they show that the use of design patterns not necessarily avoid bad smells in object oriented software system.

Our data collection was carried out by tools. The identification of design patterns was performed by DPDSS, and the identification of bad smells by RAFTool. We are not able to ensure that the results of those tools are totally right. However, we chose tools already used in previous work.

To identify the main causes of the main co-occurrences of design pattern and the bad smells identified in this study, we performed a manual inspection in the classes and in the methods involved in such co-occurrences. This inspection was carried out by one of the authors of this work. Although the inspector has high level of knowledge of all the concepts involved in the analysis, the manual inspection might be error-prone. To overcome this threat, we decided to analyze a small quantity of software systems in this work.

\section{RELATED WORK}

This section presents the main previous related work regarding the identification of co-occurrence between design patterns and bad smells.

Jaafar et al. [10] investigated the existence and the impact of the static relationship between anti-patterns and design patterns in software systems. They analyzed the behavior of these relationships during software evolution. In their work, a case study was performed with open source Java systems. The authors identified that design pattern have relationship with anti-patterns and that this relationship is in constant growth as the system evolves. In addition, the Command design pattern was identified as the one that presented the highest relation with the investigated anti-patterns.

Cardoso and Figueiredo [3] performed an exploratory analysis to investigate the co-occurrence of bad smells in software systems that use design patterns. Their study considered the God Class and Duplicate Code bad smells and eleven of twenty-three design patterns described by [9]. The authors extracted the information of design patterns and bad smells instances, and through of association rules they found the co-occurrence between Command with God Class as well as Template Method with Duplicate Code.

Jaafar et al. [11] present a study on the impact of static and co-changes dependencies in classes with design patterns and bad smells, and verify the relation of these dependencies to occurrences of software failures. In their study, the authors observed the evolution of three open-source Java software projects and concluded that classes having static dependencies with anti-patterns as well as classes having static and co-change dependencies with anti-patterns and design patterns tend to have more flaws.

Walter and Alkhaeir [18] investigated the relationship between design patterns and bad smells, and examined how the presence of one interacts with the presence of the other in a class. The authors carried out an empirical study with seven bad smells and nine design patterns, identified in two applications. They concluded that the presence of design patterns is linked with a small number of cases of bad smells.

In the present work, we identified other types of co-occurrences to the God Class and Long Method bad smells in relation to previous work, and discussed these cases. In addition we identified design patterns with low co-occurrence with the bad smells used in this work. Our study applied a different approach to detect bad smells. Our data rely in detection strategies to detect bad smells based in software metrics and their thresholds. These detection strategies were previously proposed and evaluated by Filó et al. $[7]$. 


\section{CONCLUSION}

In this study an evaluation of object oriented software systems that applies design patterns was carried out in order to (i) investigate if the use of GOF design patterns avoid the occurrences of the bad smells God Class and Long Method, (ii) identify possible co-occurrences of the GOF design patterns with these bad smells, and (iii) identify the main situations that lead software systems to present these co-occurrences. The study considered a sample of five software systems, of varying sizes. The main contribution of this work is that their findings may help the software engineering community in the comprehension of the internal structure of the software systems that apply design patterns.

To identify the bad smells in the software systems, we used detection strategies that are based in software metrics and that were previously proposed and evaluated in the literature. The detection strategies are based in metrics consistent with the characteristics of God Class and Long Method bad smells. Moreover, the thresholds of the metrics used in the strategies were also proposed and evaluated in previous work.

The results of this study show that the use of design patterns not necessarily avoid God Class and Long Method. We found that Composite and Factory Method are the design patterns less associated with the bad smells considered in this study. The main co-occurrences of design patterns and bad smells found in this work are Template Method $\Rightarrow$ God Class and Observer $\Rightarrow$ Long Method. In the manual inspection of the artifacts that presented these relations, we found classes with many responsibilities, complex methods and code repetition. A better planning of the software design and its evolution could avoid the occurrences of bad smells in the implementation of design patterns. In particular, such problems would be avoided by a better planning of the software design, as well as by the application of refactoring techniques during the evolution of the software system.

Another important information found is that most of the software systems analyzed in this work presents both bad smells, God Class and Long Method, in the implementation of the design pattern. The characteristics of these bad smells might be the reason of the co-occurrence of them. God Class is a class that performs a lot of work in a software system, and to accomplish all the tasks there are two not excluding possibilities: the class has many methods and/or the methods of the class implement many tasks, i.e., some or all of them are Long Methods.

As future works, it is important (i) to extend this research to a greater amount of sample in order the results can be generalized; (ii) to investigate co-occurrences of design patterns with other bad smells; (iii) to conduct an analysis of a larger sample considering type and size of the software systems would be also of help to improve the comprehension of software systems that apply design patterns.

\section{Acknowledgments}

This work was partially supported by CAPES.

\section{REFERENCES}

[1] R. Agrawal, T. Imieliński, and A. Swami. Mining association rules between sets of items in large databases. SIGMOD Rec., 22(2):207-216, June 1993.
[2] S. Brin, R. Motwani, J. D. Ullman, and S. Tsur. Dynamic itemset counting and implication rules for market basket data. SIGMOD Rec., 26(2):255-264, June 1997.

[3] B. Cardoso and E. Figueiredo. Co-occurrence of design patterns and bad smells in software systems: An exploratory study. In Brazilian Symposium on Information Systems, pages 347-354, 2015.

[4] K. A. M. Ferreira, M. A. Bigonha, R. S. Bigonha, L. F. O. Mendes, and H. C. Almeida. Identifying thresholds for object-oriented software metrics. The Journal of Systems and Software, 85:244-257, 2012.

[5] T. G. S. Filó. Identifying reference values for object-oriented software metrics. Master's thesis, UFMG, Computer Science, 2014.

[6] T. G. S. Filó, M. A. S. Bigonha, and K. A. M. Ferreira. Raftool - filtering tools for methods, classes and packages with uncommon measurements of software metrics. In WAMPS, pages 1-6, 2014.

[7] T. G. S. Filó, M. A. S. Bigonha, and K. A. M. Ferreira. A catalogue of thresholds for object-oriented software metrics. In SOFTENG, pages 48-55, 2015.

[8] M. Fowler and K. Beck. Refactoring: Improving the Design of Existing Code. Addison-Wesley, 1999.

[9] E. Gamma, R. Helm, R. Johnson, and J. Vlissides. Design Patterns: Elements of Reusable Object-oriented Software. Addison-Wesley, 1994.

[10] F. Jaafar, Y. Guéhéneuc, S. Hamel, and F. Khomh. Analysing anti-patterns static relationships with design patterns. ECEASST, 59, 2013.

[11] F. Jaafar, Y.-G. Gueheneuc, S. Hamel, F. Khomh, and M. Zulkernine. Evaluating the impact of design pattern and anti-pattern dependencies on changes and faults. Empirical Software Engineering, 21(3):896-931, 2016.

[12] M. Lanza and R. Marinescu. Object-Oriented Metrics in Practice. Springer-Verlag, 2006.

[13] R. Marinescu. Em Measurement and Quality in Object-Oriented Design. PhD thesis, University of Timisoara, 2002.

[14] B. L. Sousa, M. A. S. Bigonha, and K. A. M. Ferreira. A tool for detection of co-occurrences between design patterns and bad smells. Technical report, Programming Language Lab (UFMG), llp 001-2017, 2017.

[15] D. Speicher. Code quality cultivation. Communications in Computer and Information Science, 348:334-349, 2013.

[16] R. Terra, L. F. Miranda, M. T. Valente, and R. S. Bigonha. Qualitas. class corpus: A compiled version of the qualitas corpus. ACM SIGSOFT Software Engineering Notes, 38(5):1-4, 2013.

[17] N. Tsantalis, A. Chatzigeorgiou, G. Stephanides, and S. T. Halkidis. Design pattern detection using similarity scoring. Software Engineering, IEEE Transactions on, 32(11):896-909, 2006.

[18] B. Walter and T. Alkhaeir. The relationship between design patterns and code smells: An exploratory study. Information and Software Technology, 74:127-142, 2016. 